\section{Comment}

A number of cases of bilateral acute angle closure glaucoma associated with HIV have been reported. ${ }^{1-4}$ The mechanism of angle closure is thought to be due to anterior rotation of the irislens diaphragm at the axis of the scleral spur secondary to an anterior uveal effusion. Cyclopentolate retracts the iris lens diaphragm posteriorly and therefore opens the angle. Relative pupillary block does not appear to play a role in increasing intraocular pressure; hence conventional therapies for angle closure glaucoma are ineffective. The underlying cause for the effusions is not known. Unlike the previously reported cases, this case was complicated by the formation of bilateral cortical cataracts. The underlying cause of the cataracts is not known, but the possible mechanisms include; inflammatory, infective, ischaemic, and traumatic processes. Cataract of such rapid onset after moderate inflammation is unlikely. We did not find any infectious agents in the surgical specimens. Ischaemic changes, secondary to raised pressure, tend to be regional, limited to the anterior capsule and subepithelial cortical region (glaukomflecken). Cornea-lens touch was not evident, and unlikely in this anterior chamber configuration; there was no evidence of ciliary body lens touch on ultrasonography. YAG laser iridotomies may cause lenticular damage but the rapid onset, the generalised nature, and bilaterality makes this unlikely. Bilateral angle closure glaucoma in patients with HIV infection is a recognised phenomenon that requires dilatation with cycloplegia and aqueous suppressants to reverse the attack. Argon iridoplasty ${ }^{3}$ and drainage of the effusions ${ }^{1}$ have been advocated as alternative forms of therapy. This case illustrates that bilateral angle closure glaucoma in patients with HIV may be complicated by cataracts which are amenable to conventional cataract surgery.

We thank M Restori, department of ultrasonography, Moorfields Eye Hospital, London.

1 Ullman S, Wilson RP, Schwartz L. Bilateral angle-closure glaucoma in association with the acquired immune deficiency syndrome. Am F Ophthalmol 1986; 101: 419-24.

2 Williams AS, Williams FC, O'Donnell J]. AIDS presenting as acute glaucoma. Arch Ophthalmol 1988; 106: 311-2.

3 Koster HR, Liebmann JM, Ritch R, Hudock S. Acute angleclosure glaucoma in a patient with AIDS successfully treated with argon laser peripheral iridoplasty. Ophthalmic Surg 1990; 21: 501-2.

4 Nash RW, Lindquist TD. Bilateral angle-closure glaucoma associated with uveal effusion: presenting sign of HIV infection. Surv Ophthalmol 1992; 36: 255-8.
Department of

Ophthalmology, Royal Hallamshire Hospital, Sheffield S10 2RX

P Harvey

J F Talbot

University Department of Pathology, The Medical School, Sheffield

S10 2RX

J A Lee

J R Goepel

Correspondence to: Dr J A Lee, University Department of Pathology, Hill Road, Sheffield S10 2RX. Accepted for publication 11 April 1994

Figure 1 Global $(A)$ and high power $(B)$ views of the clinical appearance of the lesion.

\title{
Isolated xanthogranuloma of the limbus in an adult
}

\author{
P Harvey, J A Lee, J F Talbot, J R Goepel
}

A 30-year-old man presented with a yellow, painless, rapidly growing, raised nodule on the superior limbus of the right eye. Histological examination showed a xanthogranuloma. We discuss the clinical features, pathogenesis, and treatment of this rare condition.

\section{Case report}

A 30-year-old man was referred to the ophthal-

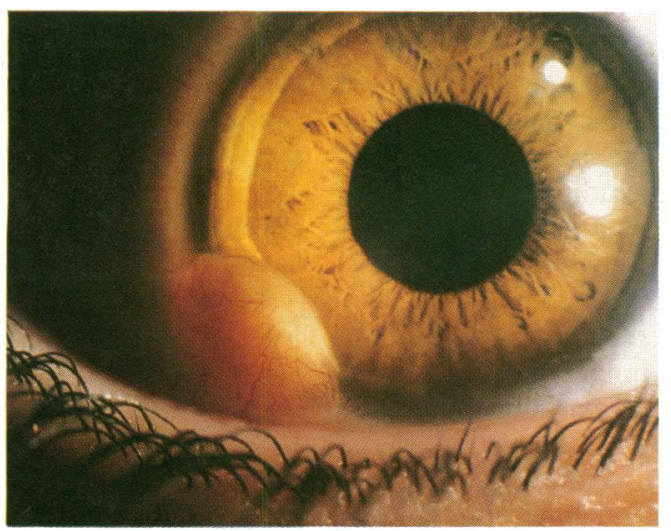

Fig IA mology clinic with a painless lesion on the superior limbus of his right eye. It had first been noticed 5 months previously, and had since rapidly increased in size and begun to encroach on the visual axis. On examination there was a well circumscribed, smooth surfaced, yellow, raised nodule at the upper temporal corneoscleral junction (Fig 1). There were no other ocular or systemic abnormalities. Excision was performed under topical anaesthesia. Surgery entailed

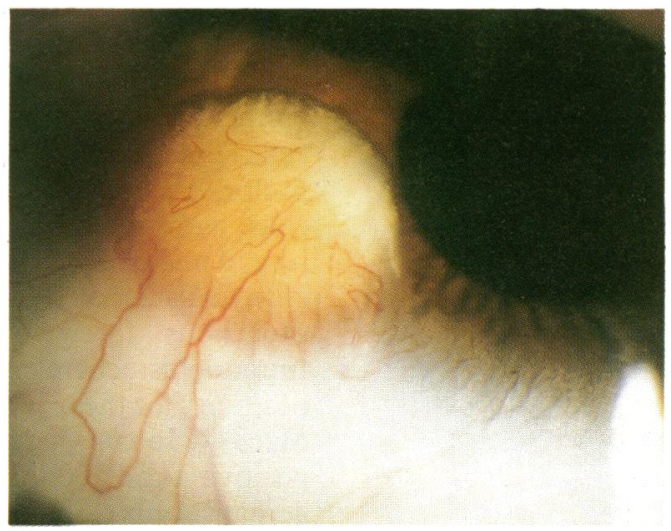

Fig IB 


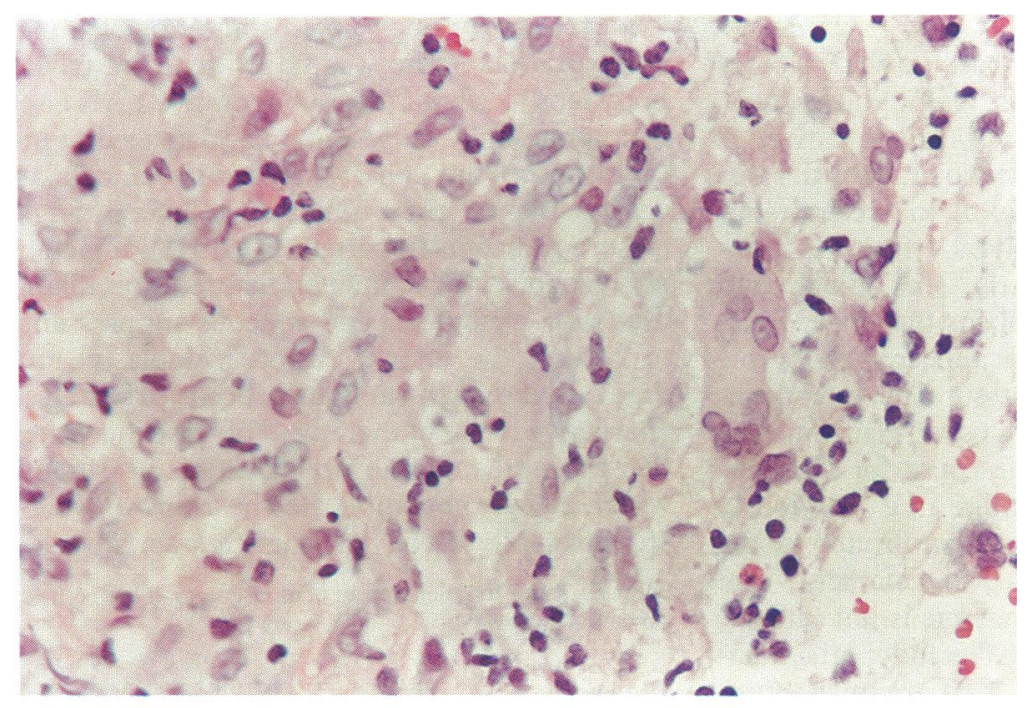

Fig $2 A$

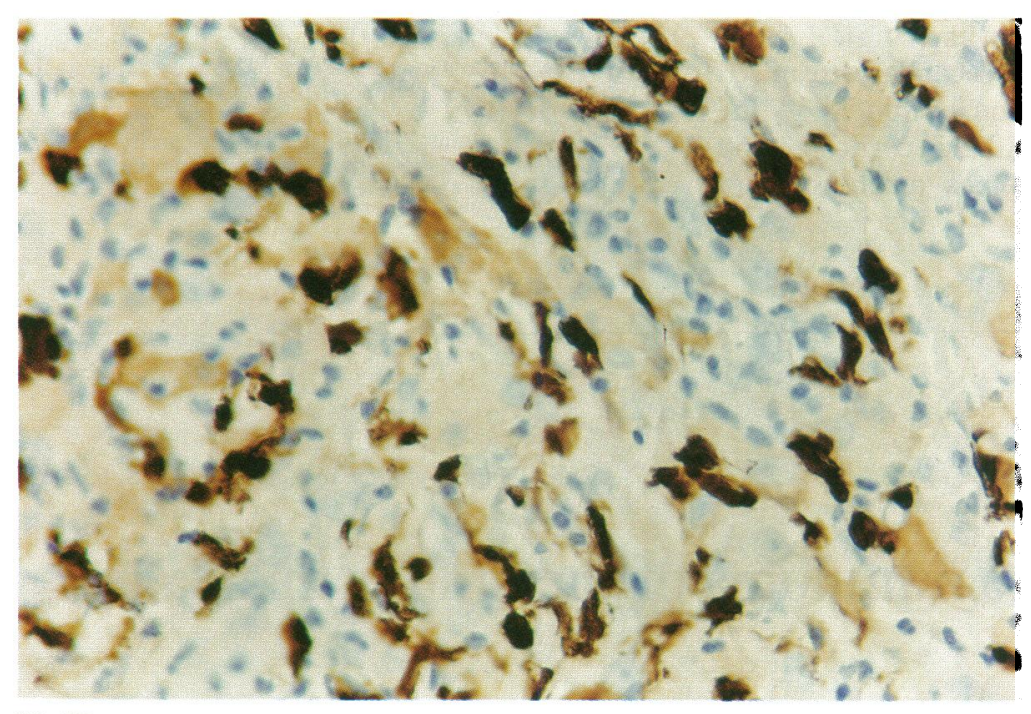

Fig 2B

Figure 2 (A)

Haematoxylin and eosin stained section showing typical appearance of juvenile xanthogranuloma. A Touton giant cell can be seen towards the right side of the field. (B) Strong positive immunohistochemical staining for the macrophage marker Mac 387. lamellar dissection of the lesion, which had penetrated approximately the anterior one third of the corneal stroma. Adjoining conjunctiva and Tenon's layer were also excised, leaving a 2-3 $\mathrm{mm}$ bare area around the site of the lesion. The patient was discharged home with antibiotic cover. Three weeks later a faint stromal scar remained and the operation site was well epithelialised. At follow up 4 months after surgery there was no sign of recurrence.

The surgical specimen consisted of an irregular fragment of tissue measuring up to $4 \times 2 \times 2 \mathrm{~mm}$. Histological examination showed normal conjunctival epithelium and oedematous underlying stroma. In the subconjunctival stroma was a nodule composed of a mixture of foamy and epithelioid histiocytes, lymphocytes, and plasma cells (Fig 2A). In addition there were scattered multinucleate giant cells, including Touton giant cells with their characteristic circumferential ring of nuclei. Special stains showed no evidence of mycobacteria, fungi, or other organisms. No exogenous foreign material was identified. Immunohistochemical staining showed strong positivity for the macrophage markers CD68 and Mac387. In contrast, only occasional cells were positive for S100 protein, a marker of Langerhans cells. Staining for factor VIII highlighted numerous small vessels in the lesion. The appearances are typical of the lesions described in juvenile xanthogranuloma.

\section{Comment}

Juvenile xanthogranuloma usually occurs in infancy, presenting at less than 1 year of age in $70 \%$ of cases. ${ }^{1}$ In these patients there are often multiple lesions, the head and neck is a favoured site and spontaneous involution before 6 years of age is common. A much smaller number of cases present in older children and adults. In these cases the lesions are usually solitary and are excised to establish a diagnosis.

In cases of typical infantile juvenile xanthogranuloma, ocular involvement is uncommon, but well recognised. ${ }^{2}$ The commonest site is the anterior uvea, but any part of the eye may be affected. Although the lesions are histologically benign, they bleed easily and are the commonest cause of spontaneous hyphaema in children. Progressive deterioration of vision caused by secondary glaucoma is a serious potential complication of uveal involvement.

Solitary xanthogranuloma affecting the corneoscleral limbus is a rare form of ocular involvement..$^{3-6}$ It is especially rare in adults, ours being apparently only the second reported case. The histological features are identical to those found in classic cases of juvenile xanthogranuloma. The lesions are distinguished from lipid granulomas because there is no extracellular lipid, from xanthomas by the presence of giant cells and from the lesions of histiocytosis $\mathrm{X}$ by lack of staining for the Langerhans cell marker $\mathrm{S} 100$ and by positivity for macrophage markers. Dermatofibroma and reticulohistiocytoma are other possible differential diagnoses, but juvenile xanthogranuloma lacks the thick, interlacing collagen fibres of the former and the large cells with glassy cytoplasm of the latter.

Clinically, bleeding does not seem to be a problem with lesions at the corneoscleral limbus, although staining with factor VIII confirmed the markedly vascular nature of the growth in this case. The main clinical problem is growth into the cornea and the necessity for a tissue diagnosis. Subconjunctival lymphoma, for example, may also present as an enlarging yellow or pink subconjunctival mass.

The aetiology remains unknown. No evidence of foreign material or an infective cause was found in the present case and none has been reported in previous cases. Nevertheless the suspicion remains that some form of local irritation is the underlying stimulus for the accumulation of activated macrophages that characterise the lesion. A previous suggestion ${ }^{3}$ that lymphostasis might be involved seems unlikely given the absence of any evidence of lymphostasis in most cases.

Several treatment modalities have been used for juvenile xanthogranuloma, including steroids, low dose radiotherapy and surgical excision. With uveal involvement non-surgical treatment is preferred because of the risk of severe bleeding. However, for limbal xanthogranuloma surgical excision would appear to be the treatment of choice and is usually curative. 
Although simple excision has been successful in some cases, ${ }^{6}$ recurrences have been reported in others. ${ }^{35}$ The latter authors recommend primary keratectomy with insertion of a lamellar graft to lessen the risk of recurrence and provide a better cosmetic result.

1 Sonoda T, Hashimoto H, Enjoji M. Juvenile xanthogranuloma: clinicopathological analysis and immunohistochemical study of 57 patients. Cancer 1985; 56: 2280-6.
2 Zimmerman LE. Ocular lesions of juvenile xanthogranuloma.

Am 7 Ophthalmol 1965; 60: 1011-35.
3 Collum LMT, Mullaney J. Adult limbal xanthogranuloma. $\mathrm{Br} \mathcal{F}$ Ophthalmol 1984; 68: 360-3.

4 Lewis JR, Drummond GT, Mielke BW, Hassard DT, Astle WF. Juvenile xanthogranuloma of the corneo-scleral limbus. Can f Ophthalmol 1990; 25: 351-4.

5 Collum LM, Power WJ, Mullaney J, Farrell M. Limbal xanthogranuloma. F Paediatr Ophthalmol Strabismus 1991; 28: 157-9.

6 Kaufman JG, Driebe WT, Hamed LM, Margo CE. Juvenile xanthogranuloma of the corneo-scleral limbus. Ophthalmic Surg 1993; 24: 428-30.

\title{
Fundus albipunctatus-like lesions in juvenile retinoschisis
}

\author{
Mary J van Schooneveld, Yozo Miyake
}

The Netherlands

Ophthalmic Research

Institute, Department of

Ophthalmogenetics,

Postbox 12141, 1100 AC

Amsterdam, the

Netherlands

$M \mathrm{~J}$ van Schooneveld

Nagoya University

School of Medicine,

65 Tsurumai-Cho,

Showa-Ku, Nagoya 466,

Japan

Y Miyake

Correspondence to:

Mary J van Schooneveld.

Accepted for publication

11 April 1994

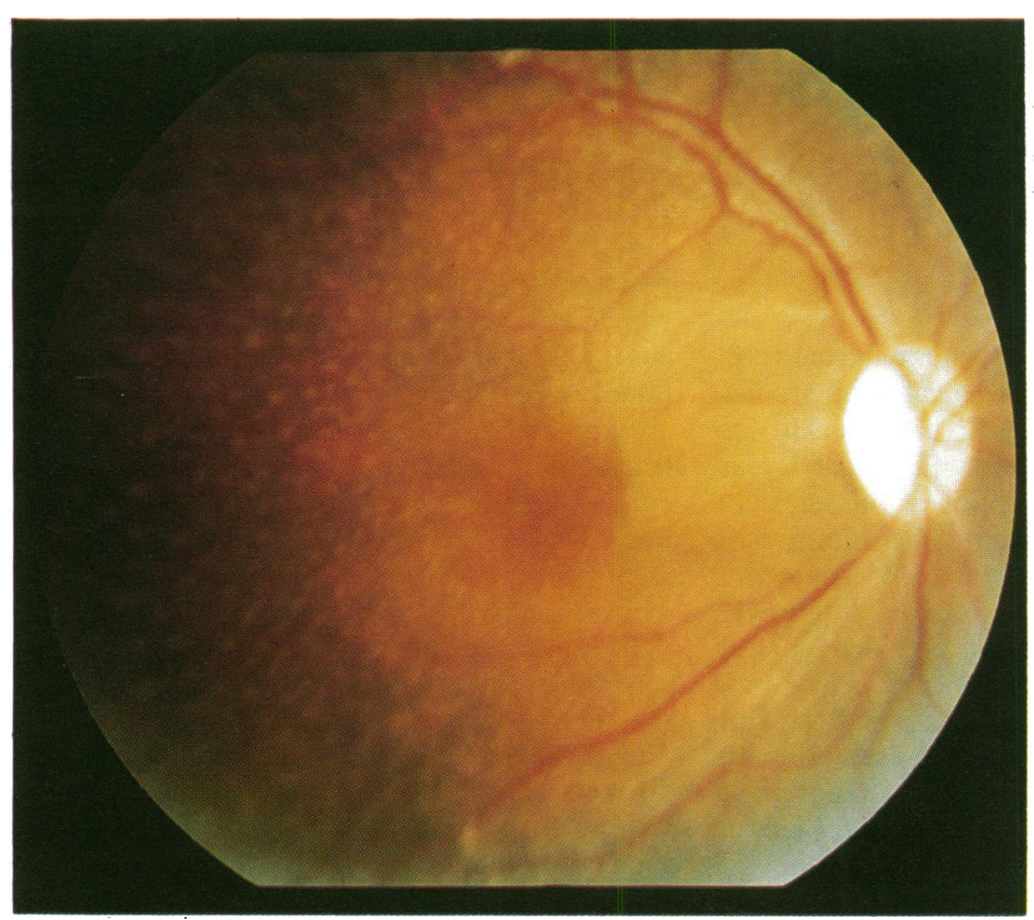

Figure $1 \mathrm{~A}$ Posterior pole in the right eye of case 1 showing cystic maculopathy and fundus albipunctatus-like lesions.

Juvenile retinoschisis (XRS) is an $\mathrm{X}$ linked vitreoretinopathy characterised mainly by typical maculopathy - namely, foveal schisis. However, the ophthalmoscopic findings may be heterogeneous, varying from a rather subtle cystic maculopathy to what may resemble an old and extensive chorioretinitis. ${ }^{1}$ Apart from the maculopathy and peripheral retinoschisis, glistening golden peripheral areas sometimes resembling the Mizuo phenomenon, dendriform lesions, neovascularisation (peripheral and of the

\section{Case reports}

CASE 1

A 10-year-old Japanese boy was diagnosed as having XRS at the age of 11 months. His maternal grandfather was also known to have this condition.

Visual acuity was 20/200 right eye with $\mathrm{S}+4 \cdot 5=\mathrm{C}+1 \times 55^{\circ}$ and $20 / 100$ left eye with $\mathrm{S}+3=\mathrm{C}+1 \cdot 25 \times 110^{\circ}$. Both fundi showed the typical star-shaped configuration in the macula and peripheral retinoschisis inferiorly. Furthermore multiple small white flecks were scattered in the temporal posterior pole (Fig 1A and 1B). These bilateral white flecks seemed to be located in the retina and not in the pigment epithelium.

The Ganzfeld electroretinogram (ERG) showed markedly reduced scotopic (rod) responses $(30-50 \%$ of normal value) and even more reduced photopic (cone) responses (20$25 \%$ of the normal value) and a negative-type ERG with a single bright flash stimulus. The oscillatory potentials were absent (Fig 2).

Visual fields showed bilateral superior loss corresponding to the schisis areas. There were no complaints of night blindness. In the 62-year-old grandfather the small flecks were absent. 